Modeling and Using Context. Third International and Interdisciplinary

Conference, CONTEXT 2001, Dundee, UK

\title{
Workflow Context as a Means for Intelligent Information Support
}

\author{
Heiko Maus \\ German Research Center for Artificial Intelligence \\ - DFKI GmbH - \\ P.O.Box 2080, 67608 Kaiserslautern, Germany \\ Heiko.Maus@dfki.de
}

\begin{abstract}
The paper presents Workflow Management Systems (WfMS) as valuable information sources for context information for accomplishing intelligent information support. First, the main advantages of WfMS for retrieving context are presented. Two projects from our research department show different approaches for retrieving workflow context and enabling an intelligent information support. However, they have to cope with the absence of a comprehensive representation concept for workflow context in WfMS. To overcome this, this paper presents a comprehensive classification scheme for workflow context.
\end{abstract}

\section{Introduction}

Systems supporting users in their daily work are becoming more and more important. There are several different approaches for information support, starting from simple web-based search engines with keyword search; continuing with systems using task-models for anticipating a user's actions and inferring relevant information [6]; up to approaches using context information from processes the user is involved in order to deliver relevant information.

In the case of business processes, a good starting point would be to consider Workflow Management Systems (WfMS) as information sources. WfMS automate processes according to process models, which define a process' activities, their order, under which conditions and when they will be carried out, by whom in the organization, with which tools, and define the 'flow' of data within the process (see $[8,11])$. A popular description is: Workflows deliver the right data to the right people with the right tools at the right time.

Because of this, WfMS seem to provide an ideal environment to retrieve a user's process context in order to deliver relevant information, thus, enabling an intelligent information support. However, which kind of context information can be delivered depends highly on the properties of the used WfMS. Unfortunately, most (commercial) WfMS do not provide such an ideal environment.

In the following, the potentials of WfMS as context providers for intelligent information support are discussed. First, the main advantages of WfMS for retrieving context are presented, including the drawbacks of current (commercial) systems. Two projects from our research department show different approaches 
for retrieving workflow context (for a detailed comparison see [3]). After that, a comprehensive classification scheme for workflow context will be presented. An overview of related work and a summary conclude this paper.

\section{WfMS as Source for Workflow Context}

Driven by discussions about context, its nature and content, e.g., at the 2000 AAAI Spring Symposium [18], and by different notions of context depending on the application domain and its usage, this paper will give insight to the notion of workflow context as we use it at our research department. As already mentioned, WfMS provide an ideal environment for retrieving workflow context. This chapter addresses the properties of WfMS which contribute to workflow context, and also points out the limitations of current WfMS. However, this section should not be an introduction to workflow management, but rather give an overview of the valuable information sources for context which a WfMS provides. It is guided by a common understanding of workflow management as expressed by the standardisation efforts of the Workflow Management Coalition (WfMC) ${ }^{1}$ [23] and several publications such as $[8,11]$.

Having a closer look on the architecture of WfMS, several sources can be found for comprising workflow context:

The process model or workflow (process) definition, specifies the constituents of the workflow. The workflow engine enacts a workflow instance according to this specification. The process model defines what, when, how, by whom, and with which data work has to be done. It consists of several objects:

- Workflow activities define how the work is actually done, e.g., by instructing the user or by invoking an application.

- The control flow defines the sequence of activity execution. Depending on the specific WfMS, several control constructs are available such as decision, alternative, parallelism, loops, start/exit conditions, or different kinds of constraints such as deadlines.

- The data flow specifies how data 'flows' through the workflow. Depending on the WfMS, different concepts exist such as input/output-container of activities or local/global variables.

The WfMC distinguishes in its glossary [23] three types of data:

workflow control data: Data that is managed by the WfMS and/or a Workflow Engine. Such data is internal to the WfMS and is not normally accessible to applications. Nevertheless, data such as instance or activity ids are usually accessible.

workflow relevant data: Data that is used by a WfMS to determine the state transitions of a workflow instance, for example within pre- and post-conditions, transition conditions or workflow participant assignment. Furthermore, this

\footnotetext{
${ }^{1}$ the WfMC is a union of workflow vendors and institutions with the goal to provide standards for workflow management, www.wfmc.org
} 
data can be typed, then the structure of the data is implied by its type and a WfMS will understand its structure and may be able to process it; and untyped - the WfMS will not understand the data structure, but may pass the data to applications.

application data: Data that is application specific and not accessible by the WfMS.

The organizational model represents the structure of an organization for declaring who actually should execute an activity. Again, depending on the WfMS, this ranges from rather simple models such as groups of users (Staffware ${ }^{2}$ ) up to complex ones with users, roles, competencies, occupation, resources, organizational hierarchy and enhanced declaration of potential workflow participants (WorkParty [16]).

The audit data represents the history of every workflow instance such as its execution log, state changes, or data changes. The audit data is an important archive for logging, performance analysis, or even legal purposes.

Considering intelligent information support, some concepts of workflow management are also worth to mention: Due to the process automation, workflows allow for invoking external applications. They provide access structures via APIs, data exchange, and even the possibility to access the workflow engine from external applications, requesting status information or control the workflow enactment. Thus, the WfMS also supports the task of information support by providing integration, monitoring, and manipulation capabilities.

Further, Jablonski et al. present in [8] causality originating from coordination theory, as a perspective worth considering in workflows, although it is not assumed essential for their workflow model. Nevertheless, causalities expressed by goals, business rules, and enterprise strategies are a valuable source for anticipating a user's behaviour or for providing information material. Therefore, causality should be considered in WfMS for intelligent information support.

Limitations with Respect to Workflow Context. Despite the existence of these various information sources, today's WfMS do not have a comprehensive representation concept for workflow context (see also [15]) and a convenient access of workflow context during runtime is not available. Therefore, one has to consider all information sources to retrieve context separately. Unfortunately, the capabilities mentioned only represent an ideal WfMS. Most of the WfMS do not provide the majority of the properties conveniently (at least from the point of view of intelligent information support). That means, some components have limited functionality as, for example, the audit data which logs only very coarse grained (e.g., start and finish of activities). Moreover, process models are often not very expressive to external applications, for instance, they only specify the control flow and a technical description of the activities to be performed, but do not provide the semantics of an activity. Therefore, an information need, necessary for information support, is hardly ever represented

\footnotetext{
${ }^{2}$ www.staffware.com
} 
To summarize, most (commercial) WfMS are not very helpful for the task of intelligent information support. But, it seems to improve due to demands resulting from introducing WfMS in heterogenous environments which require at least some powerful API. Furthermore, many research activities address limitations of WfMS (e.g., [8][7])

Considering research, a solution for intelligent information support should be generic, i.e., not depend on any specific WfMS. Despite the standardisation efforts of the WfMC, most vendors stay with their definition of workflow, their meta-models, architectures and API. Therefore, approaches trying to use context from workflows have to face several issues:

- generic vs. specific approach

- adapt workflow meta-models vs. no adaption, merely provide an add-on

- use commercial WfMS vs. research prototype

The following projects, which were conducted at our research center, show different approaches. Whereas VirtualOffice pursued a generic solution and considered only commercial WfMS, i.e., there is no chance to change any components, KnowMore built a new WfMS based on the WfMC standards, but enhanced it with concepts needed for intelligent information support.

\section{VirtualOffice : Workflow Context for Document Analysis and Understanding}

The DFKI project VirtualOffice had the goal to enhance document analysis and understanding (DAU) which deals with text recognition and information extraction from (paper) documents. DAU presents itself as a knowledge-intensive application when considering the information need of involved analysis tasks and the complexity of some application domains. Therefore, DAU systems started to also use external databases such as an address database for address verification. In addition, VirtualOffice pursued a comprehensive approach which considers all background knowledge on a document to be analysed. This knowledge can be found in a company's business processes, therefore, a generic integration into WfMS was accomplished.

The VirtualOffice-Scenario. As stated in [4], the efficiency of DAU will be significantly increased by using context information from business processes in which the documents to be analysed occur. A perfect domain for using context information is the one of business letters, which incorporates, for instance, different documents with different formats such as forms or free-form letters, with references to previous letters, open processes, or related persons. This domain distinguishes itself as having structured processes such as purchasing processes with their correspondence of orders, invoices, reminders, etc. These processes are ideal for automation by workflow management. Additionally, DAU is introduced into company's business processes placed in the business letter domain. Hence, 


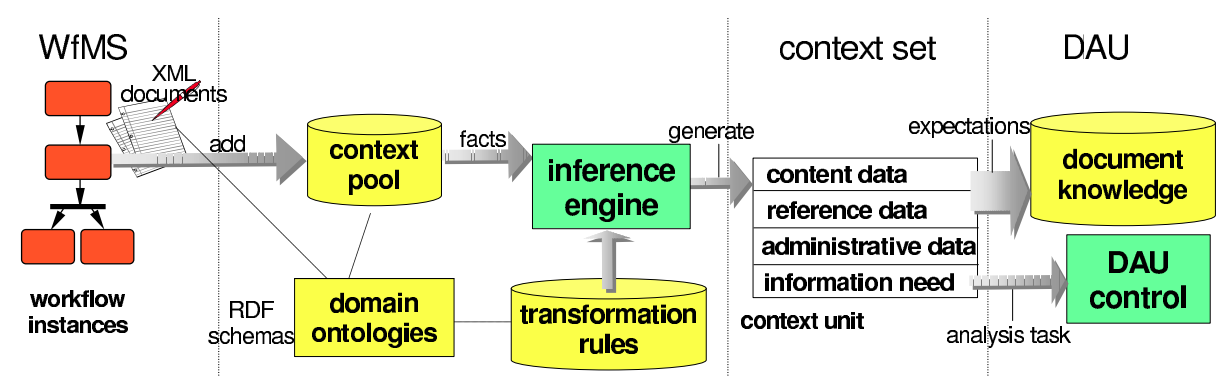

Fig. 1. Steps to retrieve context from workflows.

with an integration of both systems context information from workflows is available for supporting DAU. Because a DAU system consists of several components, each having special tasks in the overall analysis process, the components benefit from the provided context by increasing their efficiency and precision. For example, the logo recogniser can reduce its search space to those logos of companies mentioned in the context. Moreover, the pattern matching component recognises text phrases and formulations typical for distinct message types in order to classify the document and to extract the contained information. Here, by knowing which names of clerks will be mentioned in which message types, the precision can be increased and the task contributes to a identification of the corresponding workflow instance [21]. For detailed information about the VirtualOffice project, the interested reader may refer to $[22,12]$.

Retrieving Context from Workflows. The retrieval of workflow context is split into two major steps, namely context collection within workflows and context transformation into context units and storage in the context set. These steps are shown in figure 1.

As mentioned, WfMS provide several sources which allow for the collection of context information. Because VirtualOffice is supposed to provide a generic integration into WfMS independent of any proprietary extension to the workflow model, VirtualOffice has to deal with the absence of a concept for workflow context. Therefore, the so-called context pool is introduced, a database where all valuable context information considered relevant can be collected throughout the workflow execution. This ranges from single information items such as a database reference to a supplier address, up to references to involved documents such as the order. Technically, if a WfMS does not allow access to all relevant information when needed (i.e., a context unit has to be generated), then special activities are modelled in the workflow definition storing relevant information in the context pool. Thus, the context pool also serves as an archive which guarantees the availability of workflow context when needed.

Now, in case an event occurs in the workflow which will cause a response by a document (e.g., sending an order and waiting for the invoice), i.e., the workflow is waiting for a specific document, the DAU has to be informed. Therefore, the 
workflow states its expectation by generating a context unit. A context unit is generated by an inference step which uses transformation rules and the context pool as a fact base. The task of these rules is twofold: First, they derive from the available workflow context a description of the expected document. Second, they accomplish a mapping between domain ontologies used in the WfMS and the DAU's domain ontology, represented by the document knowledge. The created context unit is stored in the context set. Given this, the resulting context is more than only a collection of available information, it is rather context tailored for DAU purposes.

If a document arrives in the company's in-box, the DAU performs a process identification whereby it takes into account all expectations of all workflow instances. Once a matching expectation is found, all requested information pieces are extracted and the document is assigned to the workflow instance stated in the context unit.

Workflow Context in VirtualOffice. The necessary context information for DAU consists of a description of the document to be analysed. But, it also includes information related to the originating workflow such as id or information need. Considering this, we get a definition for workflow context for DAU purposes used in VirtualOffice: Workflow context includes all data related to a document with relevance to DAU and data required for the integration into the WfMS.

Given this definition, a context unit consists of:

content data: includes everything known about the expected document such as sender (which can be inferred form the outgoing document's recipient), file number, or product list.

reference data: includes everything which could be referenced within the expected document, such as writing date of the outgoing order, responsible clerks, and the order number.

Both represent context information for DAU purposes and are handed over as so-called expectations to the document knowledge. An expectation states content and meaning of an expected document. The DAU uses expectations within several analysis strategies: they range from a closed world assumption restricting the DAU components only to consider expectations (i.e., only results which instantiate expectations are produced), up to the generation of both, results based on expectations and results based on more general concepts from the business letter domain at the same time.

And finally, a context unit consists of:

administrative data: used for the WfMS integration, consisting of data such as workflow instance id, or the event to trigger in the workflow in case the expected document had been assigned.

information need: it states the information the workflow needs from the document for further processing, e.g., as a list of information items to be extracted. From this the DAU dynamically infers its analysis task (i.e., it does not need to extract everything). 
Besides using dynamic context from workflows, also more static context is used: so-called standard context units. These units serve two purposes: First, they describe documents not expected by a specific workflow instance such as adverts. Second, they serve as an exception handling: If there is no match between an incoming document and any context unit from the WfMS, a standard context unit is the default match (e.g., for documents of type 'invoice') and it defines the default handling, e.g., instantiation of a specific workflow definition.

VirtualOffice uses several sources for arranging its workflow context:

workflow control data: such as workflow instance id and activity id, to link a context unit to the specific instance and activity which stated it.

workflow relevant data: to fill the context pool with relevant information.

application data: also provides valuable information, e.g., the recipient's address entered into a text processor. Must also be stored in the context pool. workflow definition: The name of a workflow definition to instantiate, in case a document had been assigned to a standard context unit.

The name of an event to trigger in case the expected document is assigned.

organizational model: is used to retrieve user-specific information which could occur in a document such as the telephone number. Furthermore, can also be used in standard context units to assign documents directly to users, roles, or departments instead of instantiating a workflow definition.

information need: the information need of an activity is explicitly modelled as a list of information items needed from the document, the DAU has to extract this information. These items correspond to a domain ontology.

\section{KnowMore : Information Support for Knowledge- intensive Tasks}

In contrast to VirtualOffice, the approach of the DFKI project KnowMore (Knowledge Management for Learning Organizations) aims at the support of workflow participants dealing with knowledge-intensive tasks (kiT). With the help of workflow context, KnowMore retrieves relevant knowledge from an organizational memory (OM). This OM combines a company's information sources. The contents of these sources are modelled using formal ontologies describing conceptual knowledge.

The KnowMore -Scenario. The information support in KnowMore is a proactive (i.e., without explicit request from the user), context-sensitive delivery of relevant information. More detailed, if a workflow participant has to perform an activity which involves a kiT, the workflow engine invokes an information agent in the background. Given a kiT-description and the corresponding context from the workflow activity, the agent accomplishes an extended, ontology-based information retrieval to satisfy the user's information need by presenting relevant information (see figure 2). The information provided includes everything relevant from the company's organizational memory such as problem-related documents, 


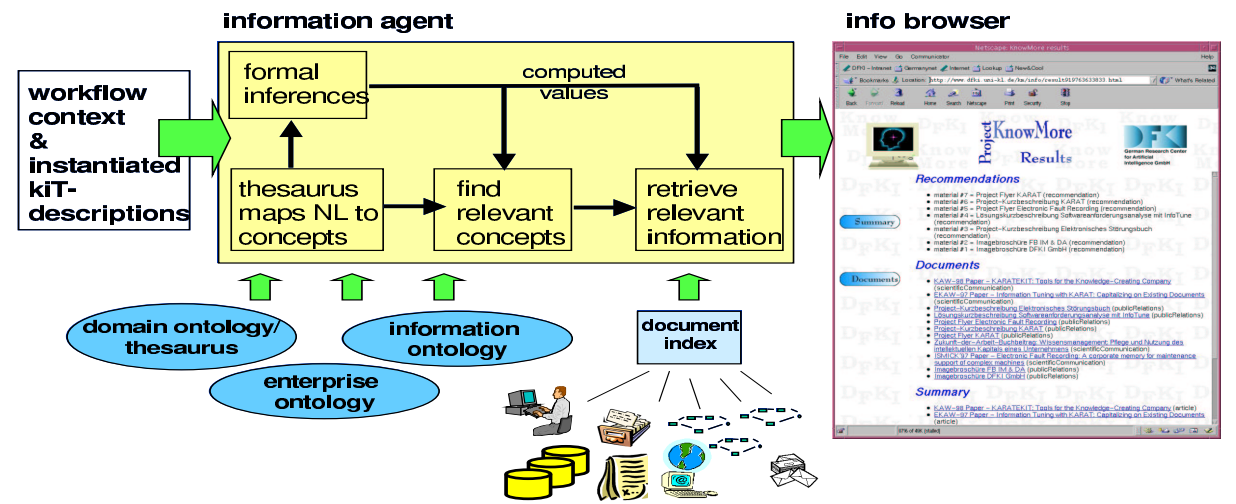

Fig. 2. KnowMore: Information Support using Workflow Context.

best practice reports, or emails from intranet discussion forums. Furthermore, references to previous workflow instances with the same problem and involved documents or information pieces. And finally, references to users involved in these workflows, or with experience because of their profile. A webpage is presented which lists links to all found information grouped by categories.

The OM considered in KnowMore contain a variety of knowledge and information sources to be searched and retrieved for information support. These sources are of different nature, resulting in different structures, access methods, and contents. To enable precise-content retrieval from heterogeneous sources, a representation scheme for uniform knowledge descriptions is needed. To this end, structure and metadata, information content, and information context are modelled on the basis of formal ontologies [2]. KnowMore uses three types of ontologies, namely

domain ontology: contains the concepts used to model the contents of information sources.

enterprise ontology: comprises the concepts used in WfMS; it is based upon the ADONIS meta-model [9] and close to the notions of the WfMC [23].

information ontology: describes the different kinds of information sources with their respective structure, access, and format properties. Furthermore, it contains generic concepts such as author, or statement types.

Retrieving Context from Workflows. In order to provide a pro-active information support, KnowMore uses an extended workflow model supporting information agents. The kiT-descriptions extend the conventional definition of a workflow activity with a support specification. It specifies the information need as generic queries together with the responsible information agent. During runtime, the agent delivers relevant information by instantiating the queries.

In order to instantiate the queries with current context, the agent must have access to workflow context suitable for the necessary reasoning for intelligent 
retrieval. But, as mentioned earlier, this goes beyond what is modelled in a conventional workflow definition. Hence, KnowMore introduces kiT-variables which are embedded into a domain ontology (i.e., their values must be of types defined as ontology concepts). The kiT-variables extend a workflow's data flow and represent the relevant context of the knowledge-intensive tasks at runtime.

Because of this, one can say that the kiT-variables extend the conventional data flow to an information flow which incorporates information pieces necessary for information retrieval. That means, the concept of workflow context is introduced by explicitly modelling the desired context in the information flow.

KnowMore also uses context-aware storage, whenever a workflow activity results in the creation of an information item worth preserving, an agent retrieves the current workflow context from all available sources such as workflow control data. Then, this context is stored in the document index and linked to the information piece to enable a context-aware retrieval later on.

Workflow Context in KnowMore. KnowMore uses several sources for its workflow context for intelligent information support, namely

workflow control data: such as workflow instance id, activity id, and current user, to link an information piece (e.g., a document) to the specific instance and activity where it was created. Furthermore, it is used within the information retrieval, e.g., to retrieve the workflow participant's profile.

workflow relevant data: KnowMore introduces kiT-variables to provide the necessary context to instantiate the generic queries.

application data: specific agents observe applications used in the workflow such as forms and, for instance, try to propose data to be filled in.

workflow definition: use of workflow definition and activity ids, to link information pieces to them. This allows for proposing related material to all instances of these definitions or activities. Moreover, activities are extended by kiT-descriptions.

audit data: used to find other workflow instances or activities with comparable kiT-descriptions

organizational model: workflow participants have competencies, interest profiles, and are part of the organizational model with its department and project hierarchies, therefore, a user-specific view on a workflow instance can be derived.

\section{A Classification Scheme for Workflow Context}

Due to the absence of a comprehensive concept for workflow context, the presented projects introduced their own solutions for retrieving workflow context. However, both use available WfMS sources.

The DFKI follow-up project Frodo (Framework for Distributed Organizational Memories, [1]) pursues - among other things - a workflow support for knowledge work. Therefore, a new WfMS will be designed which will allow us to 
overcome the limitations of current WfMS also with respect to workflow context (the vision is given in [17]).

Given all the limitations, the different architectures, and understanding of workflow, Frodo needs as a starting point a generic and extensible approach for classifying workflow context. In the following, a workflow modelling methodology will be presented, which will serve as a basis for the classification scheme.

Perspective-oriented Workflow Modelling. Jablonski et al. propose in [8] a comprehensive workflow model based on perspectives used in information system development. As a continuation, Stein proposes in [19] the corresponding methodology for developing workflow applications. These perspectives originate from methodologies of different research areas, such as business management, enterprise modelling and architecture, software process modelling, and coordination theory. The identified perspectives which are basic for the workflow model are in detail: function, behaviour, organization, information, and operation. Furthermore, but not assumed basic, history and causality. Additionally, several perspectives concerning more technical issues such as security or integrity. The different perspectives are used in the analysis and implementation phase of the workflow management application. This can be easily done, because the components of the proposed WfMS address each of these perspectives.

Workflow Context as Design Concept for WfMS. Considering the aforementioned basic perspectives including history and causality as dimensions, the required classification scheme can be derived. The resulting workflow context space is shown in figure 3 along with the properties of WfMS identified in chapter 2. Taking the perspectives as dimensions of the workflow context space provides several benefits:

- workflow context is embedded in a methodology and can be considered right from the beginning of system design.

- the specific WfMS components are responsible for different perspectives, thus, contributing to the respective dimension in the workflow context space.

- different agents need different parts of workflow context, thus, agents can easily specify the desired workflow context dimensions, therefore, enabling a more precise context delivery.

- as well as the methodology, the workflow context space stays extensible. If new perspectives are becoming important in the system, they can also be added to the workflow context space.

- the same holds the other way around: the workflow context space can be tailored to the perspectives of a specific WfMS.

The presented workflow context space serves as guideline for Frodo to consider in the WfMS architecture. That means, to enable workflow context for intelligent information support, the workflow context space will be represented as a formal ontology along with the relations to the specific components of the WfMS. Thus, all information sources of the WfMS are also accessible via the workflow context space. Furthermore, these sources will be semantically enriched by using formal ontologies. For example, the workflow definition will be repre- 


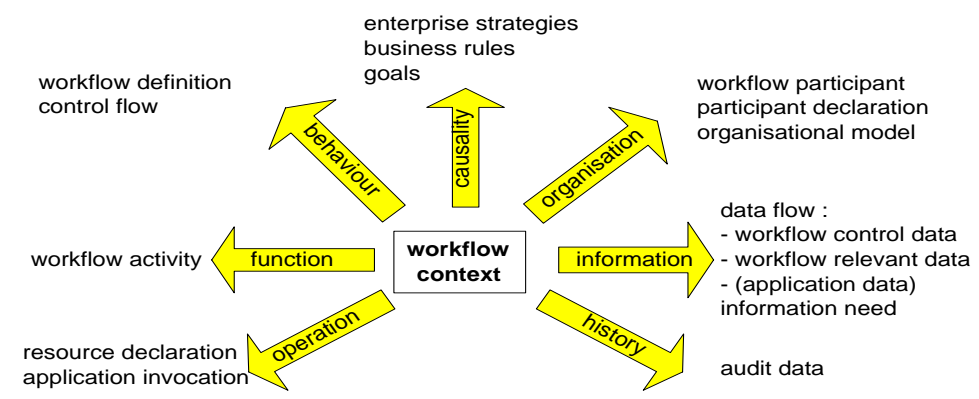

Fig. 3. The Dimensions of the Workflow Context Space.

sented using RDF (resource description framework ${ }^{3}$ ). RDF allows for expressing properties of resources, their relationships, and defining standardised models of meta-data, i.e., provides means for representing formal ontologies.

Based on this, the workflow definition can be used, e.g., to infer goals of the workflow participants. Moreover, workflow activities will be grounded in an ontology consisting of concepts describing generic tasks, thus, the activities will have some kind of semantics for external applications. Furthermore, Frodo now fully introduces the information flow concept of KnowMore. Given these properties, the audit data repository will be a valuable source for all kinds of information retrieval and for offering context information on a task based on previous workflow instances.

Furthermore, Frodo introduces a comprehensive organizational model for integration into OM, with user and role profiles, consisting of skills, experience (level of prior knowledge about a topic), and interests. These ideas are detailed in [20]. Given this, workflow context can be enriched by user-specific views on workflow instances. We believe that the Frodo WfMS will profit from this comprehensive model of workflow context.

\section{Related work}

Information Support. The Watson-system [6] is an example for a Information Management Assistant (IMA) which observes users interacting with applications and then anticipates their information needs using a task model. The Watsonsystem then retrieves documents from the internet by specifying detailed queries. Such systems could benefit from workflow context which provides an explicit process model with a user's tasks and goals. And vice versa, instead of modelling the information need during workflow design-time, as done in VirtualOffice, it could also be dynamically inferred by such IMA.

The COI IntelliDoc-system [5] provides an integration of document analysis and their WfMS BusinessFlow. In contrast to VirtualOffice, no dynamic work-

\footnotetext{
$\overline{{ }^{3} \text { www.w3c.org/RDF }}$
} 
flow context is used, but keywords occurring in specific document types. According to these keywords, documents are routed to the specified in-boxes/worklists (VirtualOffice achieves this with the standard context units).

Context from Workflows. WoMIS (Workflow Memory Information System) [7] links the WfMS CSE Workflow with a web-based information system. It organizes documents involved in the workflows, as well as supporting material (recommendations, best practice reports,...), and information on the workflow model-level (e.g., discussion about workflow definitions) in so-called contexts. A context is a tree-structure relating all involved workflow objects to information pieces such as reports about a customer to the workflow instance dealing with her credit request. The user can browse from his worklist through these contexts. New documents are manually inserted into contexts.

Context in Organizational Memories. Klemke provides in [10] a context framework for $\mathrm{OM}$ in order to identify, store, and retrieve information within $\mathrm{OM}$ according to context models. Therefore, he introduces a context agent which observes the user's current context. In case this context changes (context shift) the agent contacts a context service to retrieve relevant information according to the new context. In his context typology, he identifies three main dimensions: physical, personal, domain/content-based, and organizational. Because processes are a part of of the organizational dimension, workflow context would fit into this typology and provide additional information for other dimensions.

Context. As a representative for related work on context, we refer to Pomerol et al. who define in [14] contextual knowledge as "all the knowledge which is relevant for one person in a given situated decision problem and which can be mobilized to understand this problem and explain the choice of a given action." The rest of the context is called external knowledge. Further, they define proceduralized context as the proceduralized part of the contextual knowledge which has been invoked, structured, and situated according to a given focus. The proceduralization is triggered by an event which focused the attention of the user on parts of the contextual knowledge.

Comparing the VirtualOffice-scenario with this definition, one can say that external knowledge becomes contextual knowledge if inserted into the context pool. The context units represent parts of the contextual knowledge, because only these parts can be 'mobilized'. An event which will cause a response triggers the proceduralization (done by the inference engine) of parts of the context pool to expectations. Thus, expectations are the representation of the proceduralized context for DAU purposes.

The scenario described by Pomerol et al. is close to the one of KnowMore, because both are user centered. In KnowMore, contextual knowledge is available in the organizational memory. The generic kiT-descriptions together with the kiT-variables provide a means to obtain the necessary proceduralized context. Their instantiation and the following information retrieval represent the proceduralization. Its result is proceduralized context because the user is supported with context information relevant in her current situation. 


\section{Conclusion}

Workflow management systems automate a company's business processes. In order to achieve this, the processes have to be modelled including tasks, business rules, data, users, and applications. Because workflows are the place where a company's work is done, they are subject to various additional services such as information support. The paper pointed out that a WfMS's environment is a valuable information source and showed different components contributing to workflow context.

Two projects conducted in our research department showed in different approaches, that - although not supported by WfMS - workflow context is a reasonable concept and a valuable source for enabling intelligent information support.

However, current WfMS neither have a comprehensive representation concept for context, nor do they provide convenient access structures. Therefore, the paper presented a comprehensive approach for workflow context which builds the basis for a new WfMS designed to support knowledge work. This WfMS is supposed to overcome the limitations of current WfMS and to provide several concepts to enable workflow context as a means for intelligent information support.

Acknowledgment. This work was supported by the German Ministry for Education and Research, bmb+f (Grant: 01 IW 901, FRODO: A Framework for Distributed Organizational Memories).

\section{References}

1. A. Abecker, A. Bernardi, A. Dengel, L. van Elst, M. Malburg, M. Sintek, S. Tabor, A. Weigel, and C. Wenzel. FRODO: A Framework for Distributed Organizational Memories. Project Proposal, DFKI GmbH Kaiserslautern, 2000. URL http://www.dfki.uni-kl.de/frodo/.

2. A. Abecker, A. Bernardi, K. Hinkelmann, O. Kühn, and M. Sintek. Toward a Technology for Organizational Memories. IEEE Intelligent Systems, June 1998.

3. A. Abecker, A. Bernardi, H. Maus, M. Sintek, and C. Wenzel. Information Supply for Business Processes - Coupling Workflow with Document Analysis and Information Retrieval. Knowledge-Based Systems, Special Issue on AI in Knowledge Management, Elsevier, 13(5), 2000.

4. S. Baumann, M. Ben Hadj Ali, A. Dengel, T. Jäger, M. Malburg, A. Weigel, and C. Wenzel. Message extraction from printed documents - a complete solution. In 4th Int. Conf. on Document Analysis and Recognition (ICDAR 97), Ulm, Germany, August 18-20, 1997.

5. R. Bleisinger, M. Müller, P. Hartmann, and T. Dörstling. Intelligente Eingangspostverarbeitung mit wissensbasierter Dokumentanalyse. Wirtschaftsinformatik, (8), 1999.

6. J. Budzik and K.J. Hammond. User interactions with everyday applications as context for just-in-time information access. In Proc. of Intelligent User Interfaces 2000. ACM Press, 2000.

7. Th. Goesmann and M. Hoffmann. Unterstützung wissensintensiver Geschäftsprozesse durch Workflow-Management-Systeme. In DCSCW 2000. Springer, 2000.

8. S. Jablonski and Ch. Bussler. Workflow Management. Modeling Concepts, Architecture and Implementation. International Thomson Computer Press, 1996. 
9. D. Karagiannis, St. Junginger, and R. Strobl. Introduction to Business Process Management Systems Concepts. In B. Scholz-Reuter et al., editors, Business Process Management, LNCS. Springer, 1996.

10. R. Klemke. Context Framework - an Open Approach to Enhance Organisational Memory Systems with Context Modelling Techniques. In PAKM-00: Practical Aspects of Knowledge Management. Proc. 3rd Int. Conf., Basel, Switzerland, 2000.

11. F. Leymann and D. Roller. Production Workflow - Concepts and Techniques. Prentice Hall PTR, Upper Saddle River, New Jersey, 2000.

12. H. Maus. Towards a functional integration of document analysis in workflow management systems. In R. Becker and M. zur Mühlen, editors, Workflow Based Applications, Proc. of Workflow Management '99, Münster, Germany, 1999.

13. H.-J. Müller, A. Abecker, K. Hinkelmann, and H. Maus, editors. Workshop 'Geschäftsprozeßorientiertes Wissensmanagement' at WM'2001, Baden-Baden, Germany. http://sunsite.informatik.rwth-aachen.de/Publications/CEUR-WS/Vol-37/, 2001.

14. J. Pomerol and P. Brezillon. Dynamics between contextual knowledge and proceduralized context. In P. Bouquet et al., editors, Modeling and Using Context. Proceedings CONTEXT'99, Trento, Italy, number 1688 in LNAI. Springer, 1999.

15. U. Remus and F. Lehner. The Role of Process-oriented Enterprise Modeling in Designing Process-oriented Knowledge Management Systems. In [18], 2000.

16. W. Ruppietta and W. Wernke. Umsetzung organisatorischer Regelungen in der Vorgangsbearbeitung mit WorkParty und ORM. 1994.

17. S. Schwarz, A. Abecker, H. Maus, and M. Sintek. Anforderungen an die WorkflowUnterstützung für Wissensintensive Geschäftsprozesse. In [13], 2001.

18. St. Staab and D. O'Leary. AAAI Spring Symposium: Bringing Knowledge to Business Processes. 2000. http://www.aifb.uni-karlsruhe.de/ sst/Research/Events/sss00/.

19. K. Stein. Integration von Anwendungsprozeßmodellierung und WorkflowManagement. PhD thesis, Department of Computer Science, University of Erlangen-Nürnberg, July 1999.

20. L. van Elst and A. Abecker. Integrating Task, Role, and User Modeling in Organizational Memories. In 14 Int. FLAIRS Conference, Special Track on Knowledge Management, Key West, Florida, USA, May 2001.

21. C. Wenzel. Integrating information extraction into workflow management systems. In Natural Language and Information Systems Workshop (NLIS/DEXA 98), Vienna, Austria, 1998.

22. C. Wenzel and H. Maus. Leveraging corporate context within knowledge-based document analysis and understanding. Int. Journal on Document Analysis and Recognition, Special Issue on Document Analysis for Office Systems, 2001.

23. WfMC. Terminology and Glossary, issue 3.0. Technical report, Workflow Management Coalition, 1999. 